\title{
CARDIC MRI QUANTIFICATION OF PDA SHUNT VOLUME IN NEWBORNS
}

K.M. Broadhouse, A.N. Price, G. Durighel, D.J. Cox, A.E. Finnemore, A.D. Edwards, J.V. Hajnal, A.M. Groves

Centre for the Developing Brain, Imperial College London and MR Clinical Sciences Centre, London, UK

Background: Echo is unable to quantify volume of patent ductus arteriosus(PDA) shunt and has limited repeatability for assessing systemic perfusion. Phase Contrast MR(PCMR) can reliably assess left ventricular output(LVO) and systemic perfusion(superior vena cava(SVC), descending aorta(DAo)) ${ }^{1}$. Our aim was to use PCMR to assess LVO and total systemic flow (TSF)(as SVC+DAo-Azygous flow) to infer PDA shunt volume.

Method: PCMRI sequences(resolution-0.6/0.6/4mm,TR/TE-5.9/3.1ms) were performed to assess LVO, SVC and DAo flow at the level of the aortic valve, pulmonary trunk and diaphragm respectively. To compensate for variable contribution to SVC flow from lumbar/pelvic veins draining to the azygous system, azygous flow was quantified at the level of the diaphragm and subtracted from SVC flow.

Results: 44 infants(median weight 1755(610-3760)grams, gestation 33.7(26-39)weeks) were scanned. In 40 infants without PDA, TSF closely matched LVO(mean difference $+1 \mathrm{ml} / \mathrm{kg} / \mathrm{min}$, limits of agreement-30.3$+32.4 \mathrm{ml} / \mathrm{kg} / \mathrm{min}$ )(Figla). 4 infants had PDA shown on echo-3 infants had small, constricting ducts, 1 infant had a large PDA $(2.4 \mathrm{~mm}$, reversed diastolic DAo flow). The infant with large PDA had dramatically increased LVO $(1 \mathrm{a} / \mathrm{b})$ with PDA shunt volume estimated at $62 \%$ of LVO. However all 4 infants still had normal SVC and DAo flow(figure1c/d).
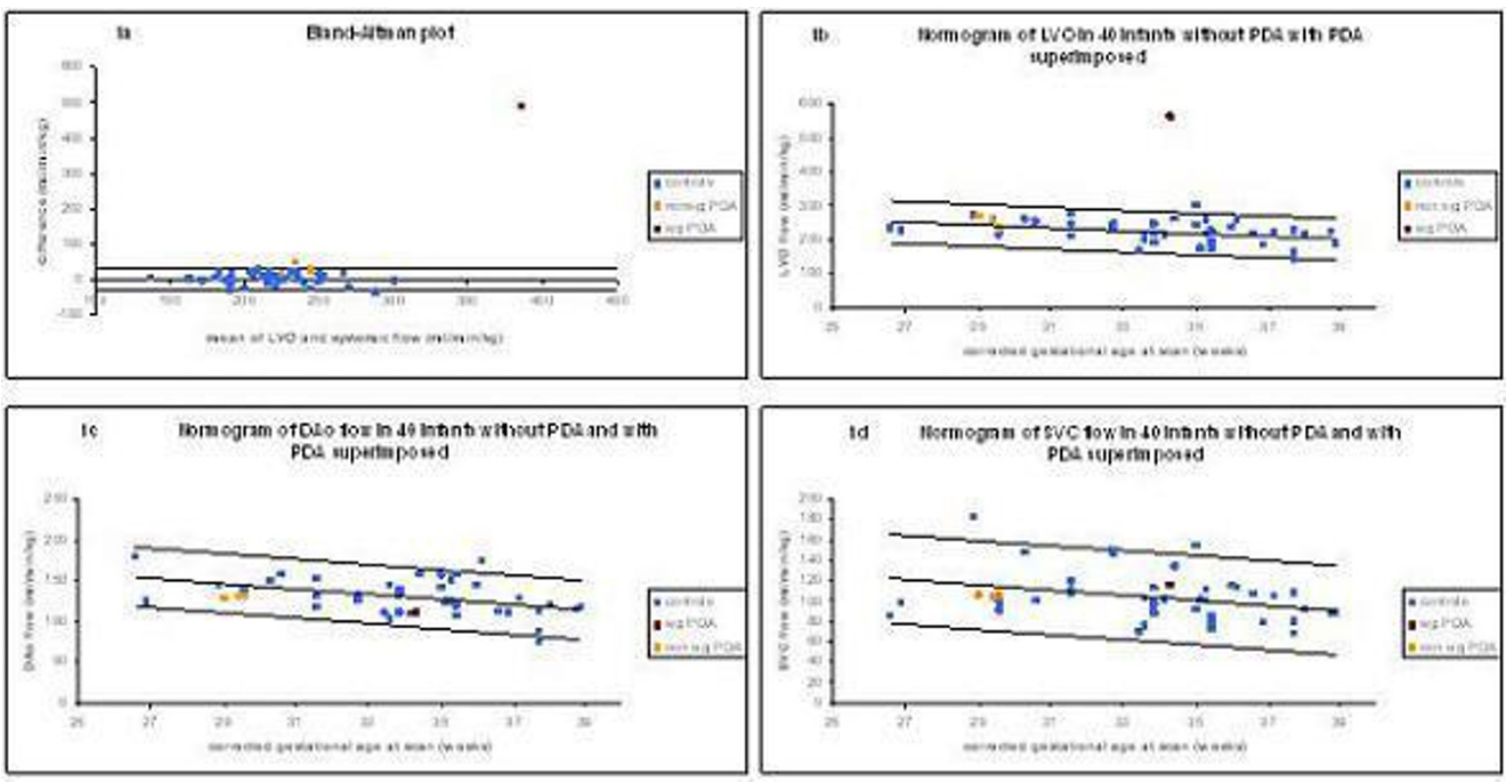

[results]

Conclusion: PCMR can reliably quantify LVO and TSF, allowing estimation of PDA shunt volume. High volume ductal shunt may not necessarily compromise systemic perfusion.

1 Groves. Arch Dis Child Fetal Neonatal Ed. Epub 2010 